\title{
Efficient Myocardial Segmentation using Local Phase Quantization (LPQ) and Automatic Segmentation Technique
}

\author{
Gayathri A. \\ Research Scholar \\ Department of computer science \\ PSGR Krishnammal College for Women, \\ Coimbatore, India
}

\author{
R. Kavitha \\ Assistant Professor \\ Department of computer science \\ PSGR Krishnammal College for women, \\ Coimbatore, India
}

\begin{abstract}
The low and high arrhythmic risk of myocardial infarction is classified based on size, location, and textural information of scarred myocardium. These features are extracted from late gadolinium (LG) enhanced cardiac magnetic resonance images (MRI) of post-MI patients. The risk level caused by features are evaluated by using various classifiers including $\mathrm{k}$ nearest neighbor (k-NN), support vector machine (SVM), decision tree, and random forest classifier. Here, high risk patients are separated from low risk patients based on the decision made by Left Ventricular Ejection Fraction (LVEF) and biomarkers based on scar characteristics. However, additional image processing techniques are needed to have clear visibility for differentiating scar texture between two risk groups. In order to maintain balanced risk groups, synthetic minority over-sampling technique (SMOTE) is used in existing system. But accuracy is limited further because of imbalance risk groups and manual segmentation of classifier. So to improve accuracy, proposed method uses automatic segmentation and Local Phase Quantization (LPQ)
\end{abstract}

\section{General Terms}

k-nearest neighbor (k-NN), support vector machine (SVM), decision tree, random forest classifier and Local Phase Quantization (LPQ).

\section{Keywords}

LG, MRI, myocardium, SMOTE, LPQ, LBP and etc.

\section{INTRODUCTION}

The medical images are taken with quality to detect the severity of the disease. Heart is the important part of human body which purifies and circulating to whole body. Heart disease is one of the most leading issues of death. Hence to predict this disease in advance, early detection and diagnosis is required [1-3]. This plays a major role in disease severity identification, predicts the outcomes of diseases and also improves patient management. Though there are several cardiac imaging modalities used for this purpose, lessinvasive imaging modalities like coronary CT angiography, cardiac magnetic resonance imaging, cardiac radionuclide imaging such as SPECT and PET modalities are widely used for assessment of heart diseases [4,5]. This study works on applications of above mentioned imaging modalities in assessing various heart diseases and provides comparison among them

Myocardial infarction (MI) indicates death of cells in the myocardium as a result of oxygen scarcity which may lead to blockage of blood supply referred as heart attack. Because of improper functioning of scarred myocardium, patients with myocardial infarction are at high risk of arrhythmias. Primary less-invasive modalities like Echocardiography, cardiac magnetic resonance (CMR), and cardiac computed tomography (CCT) are used for cardiac imaging. There are various imaging techniques for MI. Among them, Cardiac MRI and multi-slice computed tomography were widely used in the cardiac imaging technique. Implantable cardioverter defibrillators (ICDs) have been widely used to improve the survival rate of patients with arrhythmia.

Various types of segmentation methods are used in Matlab to find the scarred tissue of the myocardium. In this work region growing method is used along with Local Phase Quantization (LPQ) to segment the affected myocardial region from CMR image.

The remaining paper is prepared as follows; Section II reveals the proposed work of myocardial segmentation. Section III gives the methodology used for feature extraction and automatic segmentation. Section IV demonstrates the result of segmentation process from CMR image. Finally section $\mathrm{V}$ describes the conclusion and future enhancement of myocardial infarction segmentation.

\section{PROPOSED WORK}

The proposed work includes following phases. The first is giving affected myocardial MRI image as input. The second is processing the input MRI image using various techniques like Gabor filter and to enhance the image. The next phase uses spectral domain feature to increase the resolution of the image and the feature extraction using SVM and KNN. Final step is the segmentation of the affected myocardium image can be done using region growing method. Local Phase Quantization (LPQ) is used to give information about the scar and its location is segmented. The following figure illustrates the proposed framework for segmentation of myocardial infarction.

\subsection{Data Collection}

Data collection process is the very important in the research work. For this research the dataset are collected from the Auckland Cardiac MRI Atlas. MRI images for 32 patients who are affected by myocardial infarction are taken and each patient has 95 images with short and long axis. Every image has various pixel level with the specific time interval. The resolution of the image is $256 \times 256$ and the size of the image is $80 \mathrm{~KB}$. 


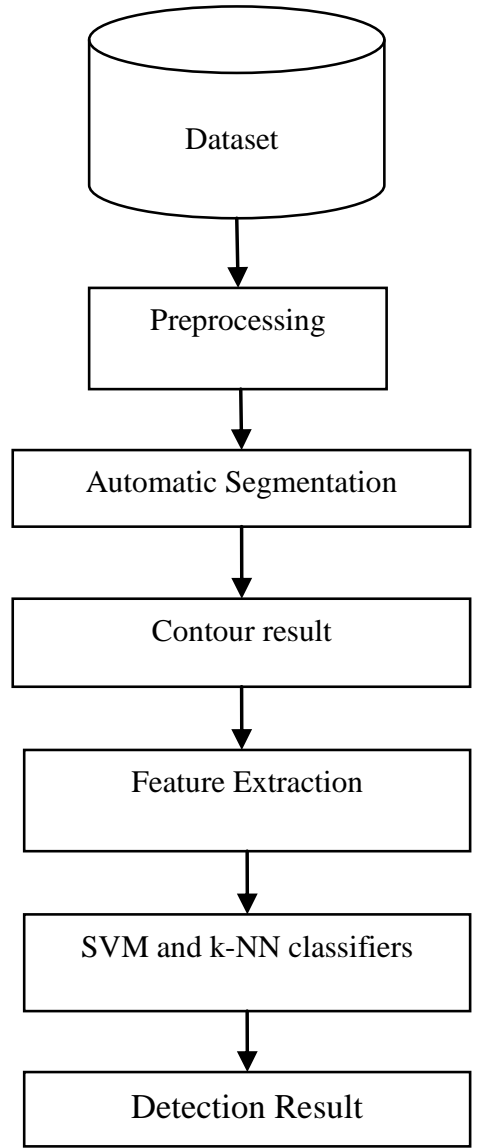

Fig1. Proposed framework for myocardial segmentation.

\subsection{Pre-Processing}

The Image data preprocessing improves the image quality by filtering and compressing the datas to minimum storage of the image and the capacity of the image during segmentation. The first step is DICOM images are converted and using the Gabor filter the magnitude of images are improved. By improving magnitude it is easy to found the scarred area of myocardium using the Local Phase Quantization(LPQ) technique.

\section{METHODOLOGY}

The methodology used to segment affected myocardium images using automatic segmentation algorithm, explained below:

\subsection{Automatic Segmentation}

Automatic segmentation method has focus on identifying the myocardial contours. In this segmentation method, 3D mesh is constructed from the cine images of patients. Then this 3D mesh is registered into the cardiac resonance image volume and rearranged to the $2 \mathrm{D}$ contours to obtain final segmentation.

\subsubsection{Template initialization:}

Myocardium is situated with Hough transform variant for annular shapes detection. Dark and circular appearance of myocardium is the reasons to detect after convolution of the image with a radially-symmetric kernel modeling a dark ring. Kernel's radial profile is given as: $f_{r}(r p)=\Delta\left(\frac{1}{\sigma \sqrt{2 \pi}} e^{-\frac{(r p-r)^{\wedge} 2}{2 \sigma^{\wedge} 2}}\right)$

Where $\mathrm{r}$ denotes the dark ring radius

\section{$\Delta$ denotes Laplacian operator}

The convolution of image is performed in the Fourier domain for different values of $r$ and selects the best solution that gives centre and radius of deformable template.

\subsubsection{Template description}

Here, myocardium seems to be a closed ribbon like structure which has image center line CL(i) $=(x(i), y(i))$ and $w(i)$ as variable width. Both $\mathrm{CL}(\mathrm{i})$ and $\mathrm{w}(\mathrm{i})$ are continuous spline interpolations of discrete set of samples given by, $\left\{\mathrm{p}_{\mathrm{s}}=\right.$ $\left.\left(\mathrm{x}_{\mathrm{S}}, \mathrm{y}_{\mathrm{S}}, \mathrm{w}_{\mathrm{S}}\right)\right\}$. Then at each node, continuous spline interpolations of a discrete set is defined as $\left\{\mathrm{p}_{\mathrm{k}}=\left(\mathrm{x}_{\mathrm{k}}, \mathrm{y}_{\mathrm{k}}, \mathrm{w}_{\mathrm{k}}\right)\right\}$. This provides a coupling between inside contours $\mathrm{C}_{\text {end }}$ (endocardium) and outside contours $\mathrm{C}_{\mathrm{epi}}$ (epicardium). The two regions such as myocardium $\left(\mathrm{R}_{\mathrm{myo}}\right)$ and blood pool $\left(\mathrm{R}_{\mathrm{bp}}\right)$

\subsubsection{Template deformation}

The criteria of set of parameters used to match template and the image evidence should be found. The input images are converted into mathematical terms expressing shape, contour and region constraints. Let $i$ represents an image and $\lambda_{i}{ }^{\prime}$ be the scalar weights balancing the various terms.

The problem is now formalised as:

$$
\begin{aligned}
& \min (p)\{f(p, i)= \\
& f_{\text {shape }}(C, w)+f_{\text {contour }}\left(C_{\text {end }}, C_{\text {epi }}, i\right)+ \\
& f_{\text {region }}\left(R_{\text {myo }}, R_{\text {bp }}, i\right)
\end{aligned}
$$

Where $f_{\text {shape }}(C, w)$ represents the shape and it is expressed by,

$$
\begin{aligned}
& f_{\text {Shape }}(C, w)= \\
& \lambda_{0} \int_{0}^{1}|\operatorname{avg}(C L(i))-\overline{\operatorname{avg}}|^{2} d C L(i)+ \\
& \lambda_{1} \int_{0}^{1} \mid w^{\prime}\left(\left.(C L(i))\right|^{2} d(C L(i)\right.
\end{aligned}
$$

Where $\operatorname{avg}(\mathrm{CL}(\mathrm{i}))$ denotes the centreline curvature with average avg.

$f_{\text {contour }}\left(C_{\text {end }}, C_{\text {epi }}, i\right)$ represents the Contour given as,

$f_{\text {contour }}\left(C_{\text {end }}, C_{\text {epi }}, i\right)=\lambda_{2} \int_{0}^{1} \nabla I_{\text {in }}(i) d i-$ $\lambda_{3} \int_{0}^{1}\left|\nabla I_{\text {in }}(i)\right| d i$

Where image gradient $\nabla I_{i n}$ is expressed as $\nabla_{\mathrm{I}}$ $\left(\mathrm{C}_{\text {end }}(\mathrm{i})\right) \cdot \mathrm{n}(\mathrm{i})$

$f_{\text {region }}\left(R_{\text {myo }}, R_{b p}, i\right)$ represents the region and it is given as, 


$$
\begin{aligned}
& f_{\text {region }}\left(R_{\text {myo }}, R_{b p}, i\right)=\frac{\lambda_{4}}{\left|R_{m y o}\right|} \int_{R_{m y o}} \mid i(x, y)- \\
& \overline{R_{\text {myo }}}\left|d x d y+\frac{\lambda_{5}}{\left|R_{b p}\right|} \int_{R_{b p}}\right| i(x, y)-\overline{R_{b p}} \mid d x d y+ \\
& \lambda_{6}\left(\overline{R_{\text {myo }}}-\overline{R_{b p}}\right)
\end{aligned}
$$

\subsubsection{Abnormal tissue detection}

Each slice of CMR volume is pre-processed before the template deformation. The intensity distribution mixture is then calculated by Expectation-Maximisation algorithm. Areas with respect to healthy myocardium and abnormal tissues are expected to be appeared as homogeneous regions of the minimum $(\overline{\mathrm{h}}(\min ))$ and maximum $(\overline{\mathrm{a}}(\max ))$ intensities in the new range. Potential scars or ischemic areas are detected by computing Brightness, Homogeneity around the expected value and Homogeneity. Thus, affected part of heart is obtained by automatic segmentation method. Then 3D mesh is constructed by computing the transformation of cine and LE CMR data and obtained the centre point of each image slice. Then axes are determined from this centre point using least mean square algorithm. Finally, the constructed mesh is deformed in which image intensity is included in the final myocardial contour.

\subsection{Feature Extraction}

Quantitative MRI features that possess information such as size, location and textures about scarred myocardium are considered and they are extracted to categorize the patients of HAG and LAG.

\subsubsection{Relative scar volume}

Scar size is generally utilized as biomarkers. First, Relative Scar Volume is determined by using Segmented CMR slices and finally gives the information about scar size. It is the ratio of number of pixels present in the scar to the number of pixels present in the myocardium, across all the CMR slices.

$$
S_{r s v}=\frac{\sum_{R=1}^{T n}\left|S_{R}\right|}{\sum_{R=1}^{T n}\left|M_{R}\right|}
$$

Where $\left|S_{R}\right|$ and $\left|M_{R}\right|$ are the number of pixels in the associated scarred and myocardial region

\section{And Tn represents the total number of slices}

\subsubsection{Localization Features}

For extracting morphological information of scarred tissue, localization features should be determined by using reference point $\mathrm{P}_{\mathrm{R}}$ on $\mathrm{CMR}$ slices. $\mathrm{P}_{\mathrm{R}}$ is estimated by considering average position of centroids of all pixels in $\operatorname{Epi}_{R}, M_{R}$ and End $_{R}$. $A_{\text {pos }}$ is an angular position which represents the angle between the line connecting the pixel to reference point $P_{R}$. then left ventricle $L V$ is drawn manually by cardiologists. The localization features with statistics such as mean, standard deviation and median are easily calculated for each pixel present in the scarred volume.

\subsubsection{Scar gradient}

Third, Infarct tissue heterogeneity for arrhythmic patients is found by determining Scar gradient. The rate of change of pixel intensity is estimated from the derivative images and they are used for the identification of Infarct tissue heterogeneity. The final feature gradient is the sum of gradient magnitude of all pixels in scarred volume and it is identified by,

$\mathrm{G}_{\mathrm{scar}}^{(\mathrm{w})}=\sum_{\mathrm{t}=1}^{\mathrm{Tn}} \sum_{\mathrm{i}, \mathrm{j}} \mathrm{M}_{\mathrm{G}}^{(\mathrm{w})}(\mathrm{i}, \mathrm{j})$

Where $\mathrm{M}_{\mathrm{G}}^{(\mathrm{w})}$ represents the gradient magnitude and it is expressed by,

$$
M_{G}^{(w)}(i, j)=\sqrt{\left[D_{G, x}^{(w)}(i, j)\right]^{2}+\left[D_{G, y}^{(w)}(i, j)\right]^{2}}
$$

Where $\mathrm{D}_{\mathrm{G}, \mathrm{X}}^{(\mathrm{w})}$ and $\mathrm{D}_{\mathrm{G}, \mathrm{y}}^{(\mathrm{w})}$ represents the directional derivative images achieved after convolution.

\subsubsection{Local Phase Quantization (LPQ)}

For accurate detection of scarred part in images, the image is quantized using Local Phase Quantization

Local Phase Quantization (LPQ) performs with respect to the blur invariance property. This method utilized the phase information extracted by applying 2-D DFT or by short-term Fourier transform (STFT) measured over image $\mathrm{f}(\mathrm{x})$ given by,

$$
F(u, x)=\sum_{y \epsilon N_{x}} f(x-y) e^{-j 2 \pi u^{T} y}=w_{u}^{T} f_{x}
$$

\section{Where}

$\mathrm{W}_{\mathrm{a}}$ is the basis vector of the 2-D DFT at frequency a

LPQ considers only four complex coefficients with respect to 2-D frequencies such as $\mathrm{a}_{1}=[\mathrm{a}, 0]^{\mathrm{T}}, \mathrm{a}_{2}=[0, \mathrm{a}]^{\mathrm{T}}, \mathbf{a}=[\mathrm{a}$, $\mathrm{a}]^{\mathrm{T}}$ and $\mathrm{a}_{4}=[\mathrm{a},-\mathrm{a}]^{\mathrm{T}}$ where a represents the scalar quantity.

$F_{x}=\left[\operatorname{Re}\left\{F_{x}^{c}\right\}, \operatorname{Im}\left\{F_{x}^{c}\right\}\right]^{\mathrm{T}}$

Where $\mathrm{F}_{\mathrm{x}}^{\mathrm{c}}=\left[F\left(\mathrm{a}_{1}, \mathrm{x}\right), F\left(\mathrm{a}_{2}, \mathrm{x}\right), F\left(\mathrm{a}_{3}, \mathrm{x}\right), F\left(\mathrm{a}_{4}, \mathrm{x}\right)\right]$

$$
F_{x}=W f_{x}
$$

Where

$\mathrm{W}=\left[\operatorname{Re}\left\{\mathrm{w}_{\mathrm{a} 1}, \mathrm{w}_{\mathrm{a} 2}, \mathrm{w}_{\mathrm{a} 3}, \mathrm{w}_{\mathrm{a} 4}\right\}, \operatorname{Im}\left\{\mathrm{w}_{\mathrm{a} 1}, \mathrm{w}_{\mathrm{a} 2}, \mathrm{w}_{\mathrm{a} 3}, \mathrm{w}_{\mathrm{a} 4}\right\}\right] T$

Then the transform coefficient vector $\mathrm{F}_{\mathrm{X}}$ is stored in covariance matrix $\mathrm{C}$. The histogram of LPQ strings are built and they are normalized to each CMR slice of image.

\subsubsection{Chi-square distance}

After histogram formation, classification scheme is adapted to classify HAG and LAG patients. For this classification, two histograms and distance metrics are required. So for measuring distance, chi-square distance is used. It works better for smaller dataset group. Learning the model histogram is done by taking the average of training histogram. Then the test patient is assigned which has minimum distance compared to the model histogram of HAG and LAG patients. Chi square distance is expressed as follows: 


$$
d^{2}\left(H_{s}, H_{m}\right)=\sum_{b=1}^{B} \frac{\left(H_{s b}-H_{m b}\right)^{\wedge} 2}{\left(H_{s b}+H_{m b}\right)}
$$

Where $\mathrm{H}_{\mathrm{sb}}$ represents the frequencies of same bin $\mathrm{b}$ in $\mathrm{H}_{\mathrm{s}}$

$\mathrm{H}_{\mathrm{mb}}$ represents the frequencies of same bin $\mathrm{b}$ in $\mathrm{H}_{\mathrm{m}}$

Finally, the extracted scalar features are used in k-NN and SVM classifiers. This is defined as:

$D_{P, R}=\frac{d^{2}\left(H_{S}, H_{m}{ }^{H}\right)}{d^{2}\left(H_{S}, H_{m}{ }^{L}\right)}$

Where $\mathrm{H}_{\mathrm{S}}$ represents the patients of LPQ histogram

$\mathrm{H}_{\mathrm{m}}{ }^{\mathrm{H}}$ and $\mathrm{H}_{\mathrm{m}}{ }^{\mathrm{L}}$ represents the patients in model histograms of $\mathrm{HAG}$ and $\mathrm{LAG}$

But for high risk arrhythmic patients, large values of chisquare features are needed.

\section{RESULTS}

The effective myocardial infarction segmentation is implemented in Matlab R2014a software. The CMR images are segmented using Local Phase Quantization (LPQ) and automatic segmentation.

\subsection{Noise Removal}

CMR images are filtered to remove the noise based on intensity values of the pixels using the Gabor filter to improve the magnitude of image using Matlab. The figure (fig2) shows the original image and the filtered image.

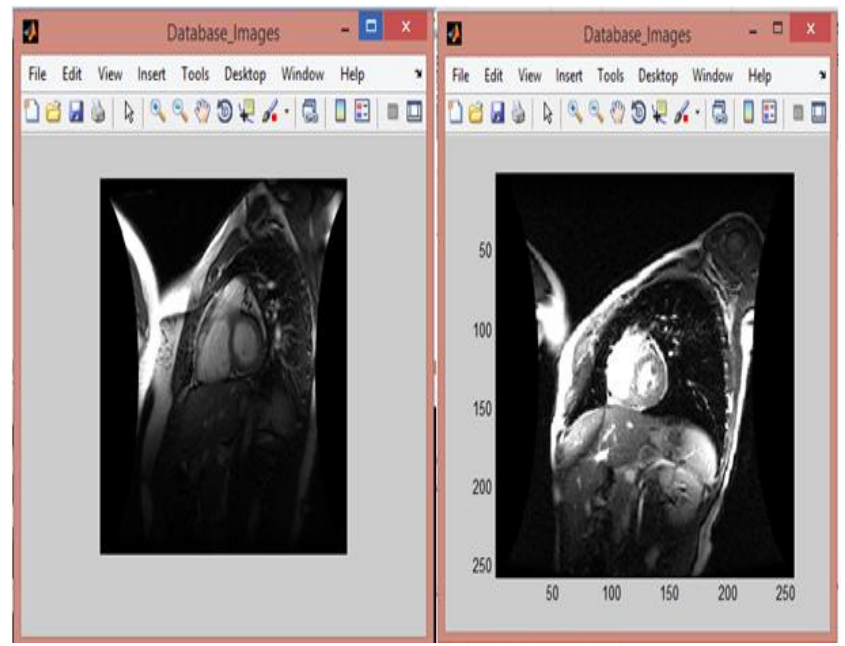

Fig1. Noise Removal using Gabor filter

\subsection{Automatic Segmentation}

In the automatic segmentation various algorithms are used and the affected regions are partitioned using contour image and segmented.

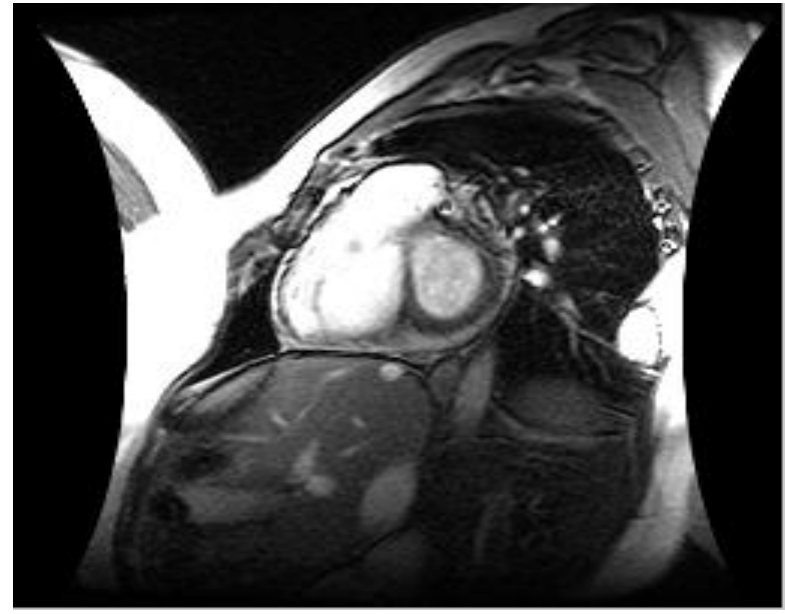

Fig2. Input image

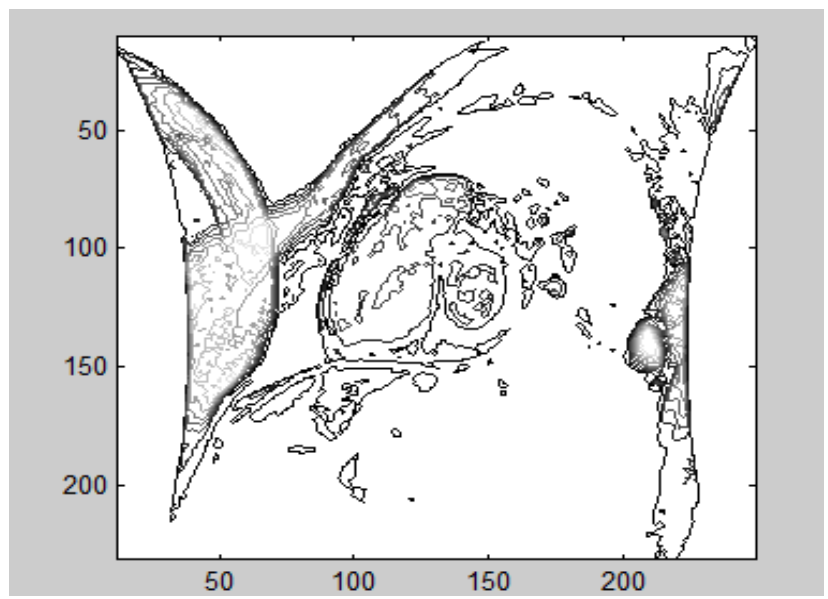

Fig3. Contour image

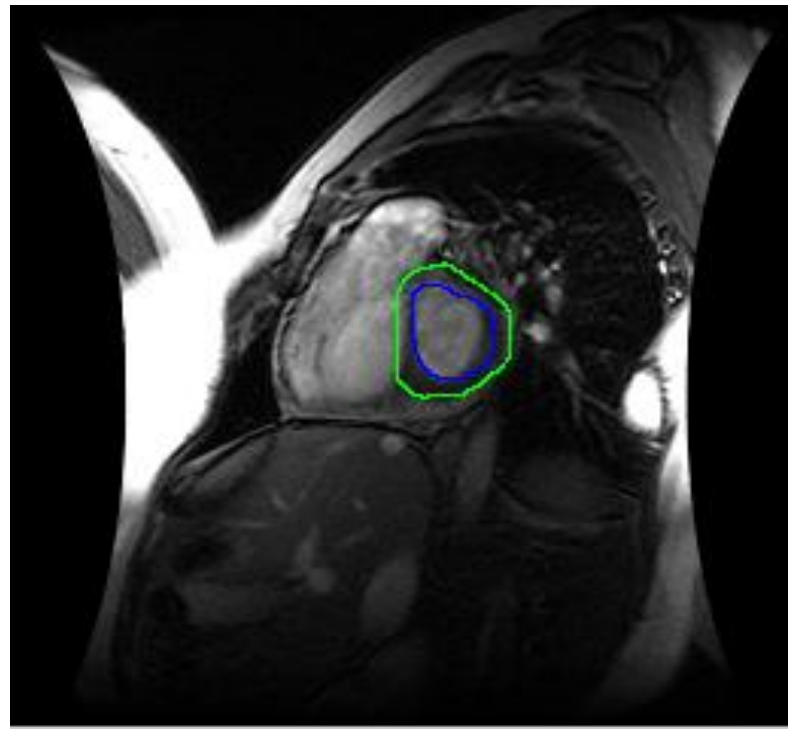

Fig4. Segmented image 


\subsection{Performance Evaluation}

\subsubsection{Classification Accuracy}

The below fig1 shows the classification accuracy result of existing LBP with segmentation method and proposed LPQ based automatic segmentation method. It should be noted that, Scarred features that are obtained from LPQ improves the performance of both SVM and k-NN classifiers.

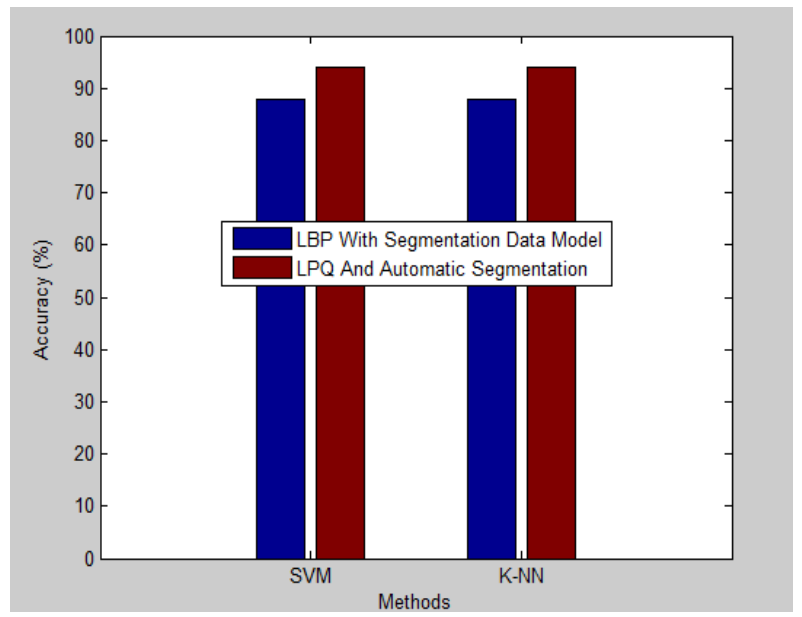

Fig1. Accuracy result of LBP method and LPQ based automatic segmentation method

\subsubsection{Precision}

The below fig2 shows the precision result of existing LBP with segmentation method and proposed LPQ based automatic segmentation method. LPQ method accurately predicts the error and enhances the precision result of both SVM and k-NN classifiers.

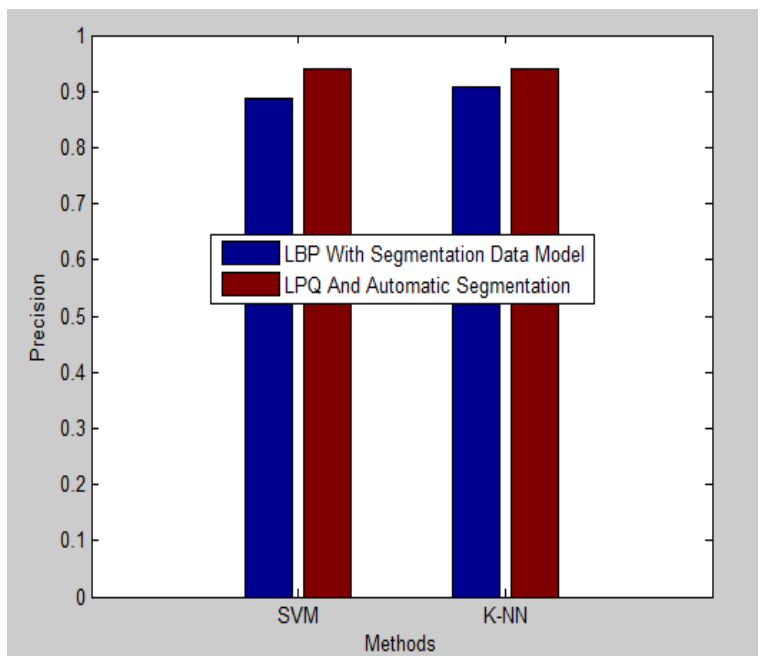

Fig2. Precision result of LBP method and LPQ based automatic segmentation method

\subsubsection{Recall}

The below fig 3 shows performance result on recall of existing LBP with segmentation method and proposed LPQ based automatic segmentation method. Automatic segmentation and LPQ method both increases the prediction of relevant features in large data set and improves the classification performance.

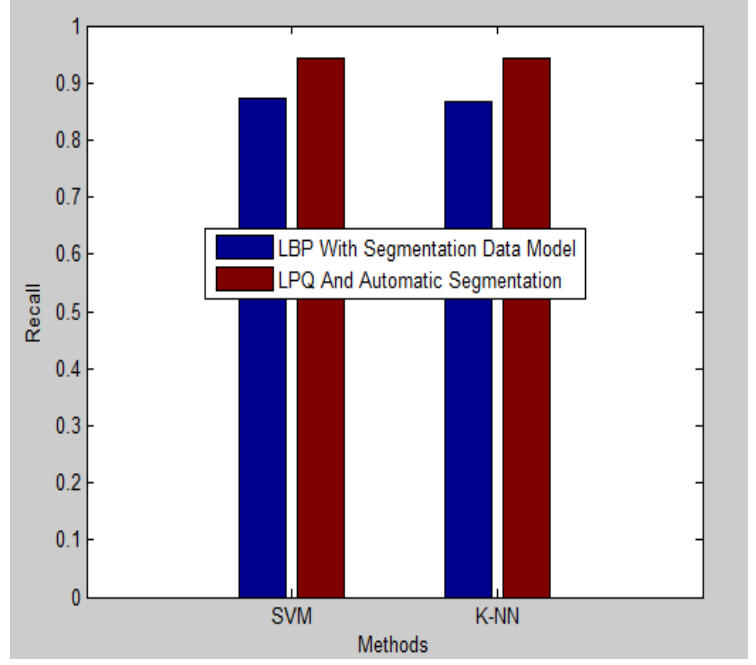

Fig3. Recall result of existing method and LPQ based automatic segmentation method

\subsubsection{F-Measure}

The below fig 4 shows the result of F-measure of LBP with segmentation method and LPQ based automatic segmentation method. From the figure, it is proved that, proposed system with automatic segmentation and LPQ outperforms than the existing system.

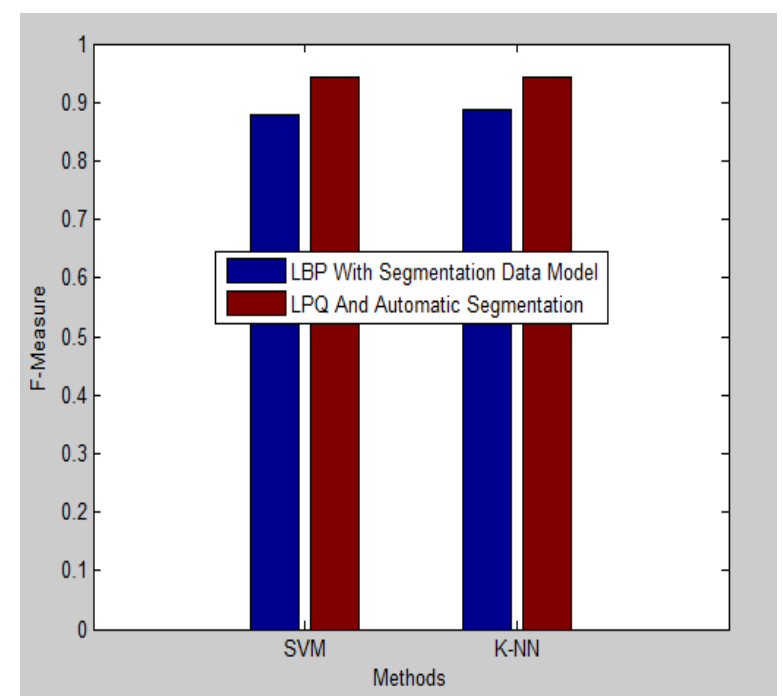

Fig4. F measure comparison of LBP method and LPQ based automatic segmentation method

\section{CONCLUSION}

In this research the MRI myocardial images were segmented to detect the myocardial infarction between low and high arrhythmic groups. The feature extraction is done to improve the accuracy. LPQ technique is used to give the information about the scarred tissue such as size of the scar and where the scar is located. In future research, the proposed algorithm may test using larger number of datasets and alternative methods are used to improve the accuracy. Various algorithms may be used for detecting various myocardial diseases. 


\section{REFERENCES}

[1] Issac N.Bankman, "Hand Book of medical image processing and analysis", Elsevier Inc. , Isbn 978-0-12 $373904-9,2^{\text {nd }}$ edition, 2009 .

[2] B. Chanda, D. D. Majumder, Digital Image Processing and Analysis, PHI Learning Private Limited, New Delhi, Isbn 978 -81-203 -4325-2, 2nd Edition, 2011.

[3] Palaniappan. S, Awang.R, "Intelligent heart disease prediction system using data mining techniques". In Computer Systems and Applications, AICCSA,IEEE/ACS International Conference on(pp. 108-115), 2008.

[4] Ali, M. T., \& Sabri, S. "Utility of cardiac MRI in the assessment of myocardial viability evaluating its role using 3-Tesla machine in correlation with SPECT", 2016.

[5] Knaapen, P., De Haan, S., Hoekstra, O. S., Halbmeijer, R., Appelman, Y. E., Groothuis, J. G. J., \& Götte, M. J. W, "Cardiac PET-CT: advanced hybrid imaging for the detection of coronary artery disease" Netherlands Heart Journal, 18(2), 90-98,2010.

[6] Jabbar, M. A., Chandra, P., \& Deekshatulu, B. L, "Prediction of risk score for heart disease using associative classification and hybrid feature subset selection”, In ISDA (pp. 628-634), 2012.

[7] Alizadehsani, R., Habibi, J., Hosseini, M. J., Mashayekhi, H., Boghrati, R., Ghandeharioun \& Sani, Z. A, " A data mining approach for diagnosis of coronary artery disease" Computer methods and programs in biomedicine, 111(1), 52-61, 2013.
[8] Grbic, S., Ionasec, R., Mansi, T., Georgescu, B., VegaHiguera, F., Navab, N., \& Comaniciu, D, "Advanced intervention planning for Transcatheter Aortic Valve Implantations (TAVI) from CT using volumetric models", IEEE 10th International Symposium on Biomedical Imaging (pp. 1424-1427), 2013.

[9] Jabbar, M. A., Deekshatulu, B. L., \& Chandra, P, "Heart disease prediction using lazy associative classification", In Automation, Computing, Communication, Control and Compressed Sensing (iMac4s), International MultiConference on (pp. 40-46). IEEE, 2013.

[10] Sonawane, J. S., \& Patil, D. R, "Prediction of heart disease using learning vector quantization algorithm", In IT in Business, Industry and Government (CSIBIG), (pp. 1-5). IEEE, 2014.

[11] Ilayaraja, M., \& Meyyappan, T, "Efficient Data Mining Method to Predict the Risk of Heart Diseases Through Frequent Itemsets", Procedia Computer Science, 70, 586-592,2015.

[12] Jonnagaddala, J., Liaw, S. T., Ray, P., Kumar, M., Chang, N. W., \& Dai, H. J, "Coronary artery disease risk assessment from unstructured electronic health records using text mining", Journal of biomedical informatics, 58, S203-S210, 2015.

[13] Lakshmi, K. P., \& Reddy, C. R. K, "Fast rule-based heart disease prediction using associative classification mining", In Computer, Communication and Control (IC4), International Conference on (pp. 1-5),IEEE,2015. 